\title{
Solving differential equations for Feynman integrals by expansions near singular points
}

\section{Roman N. Lee}

Budker Institute of Nuclear Physics, 630090 Novosibirsk, Russia

E-mail: roman.n.leedgmail.com

\author{
Alexander V. Smirnov \\ Research Computing Center, Moscow State University, 119991, Moscow, Russia \\ E-mail: asmirnov80egmail.com
}

\section{Vladimir A. Smirnov*}

Skobeltsyn Institute of Nuclear Physics of Moscow State University, 119991, Moscow, Russia

E-mail: smirnovethoery.sinp.msu.ru

\begin{abstract}
We advocate a strategy to solve differential equations for Feynman integrals by powers series expansions near singular points and to obtain high precision results for the corresponding master integrals. We consider Feynman integrals with two scales, i.e. nontrivially depending on one variable. The corresponding algorithm is oriented at situations where canonical form of the differential equations is impossible. We provide a computer implementation of the algorithm in a simple example of four-loop generalized sun-set integrals with three equal non-zero masses. Our code provides values of the master integrals at any given point on the real axis with a required accuracy and a given order of expansion in the regularization parameter $\varepsilon$.
\end{abstract}

13th International Symposium on Radiative Corrections

24-29 September, 2017

St. Gilgen, Austria

\footnotetext{
* Speaker.
} 


\section{Introduction}

Evaluating Feynman integrals with differential equations (DE) initiated in [1,2] and formulated as a method to evaluate master integrals in [3-6] became one of the most powerful methods. Still this method is under development. In [7] it was suggested to turn from the basis of primary master integrals (i.e. revealed when solving integration by parts relations [8]) to the so-called canonical basis for which the right-hand side of the of system of DE is proportional to $\varepsilon=(4-D) / 2$ and the singularities of the matrix on the right-hand side of DE are Fuchsian. The first algorithm to arrive at the canonical form was constructed in the case of one variable in Ref. [9] (Such form of $\mathrm{DE}$ was called $\varepsilon$-form there). Besides the private implementation of this algorithm by its author and several other private implementations, two public implementations, Fuchsia $[10,11]$ and epsilon [12], of the algorithm of Ref. [9] are now available. ${ }^{1}$

Once DE for master integrals are converted into an $\varepsilon$-form, i.e. one finds an appropriate linear transformation to a canonical basis, solving DE becomes straightforward, order-by-order in $\varepsilon$. Typically, the corresponding results are expressed naturally in terms of harmonic polylogarithms [15] or multiple polylogarithms [16]. These functions are very well studied. For harmonic polylogarithms, one can apply the package HPL [17] which encodes various analytical properties and provides the possibility of numerical evaluation with a very high precision. For multiple polylogarithms, one can use the computer implementation [18] of the algorithm GiNaC [19] to obtain high-precision numerical values, up to several thousand digits and more.

It is well known that the $\varepsilon$-form of DE for a given set of the master integrals is not always achievable by rational transformations. For massive internal lines it is often required to consider also transformations involving square roots. However, even using transformations from this extended class it is not always possible to obtain an $\varepsilon$-form. ${ }^{2}$ The simplest example where an $\varepsilon$-form is impossible is given by the two-loop propagator sunset diagram with three identical masses. In this example, as well in other known examples without $\varepsilon$-form, DE can still be reduced to the form where the right-hand side of the differential system is a linear function of $\varepsilon$.

However, 'integrating out' the constant term in such a form of DE appears to be an essentially more complicated problem. This can be seen in the known examples where results are expressed in terms of elliptic functions. In practice, it can happen that such 'elliptic' master integrals appear only in a small number of sectors. (A sector is specified by a distribution of the set of indices (powers of propagators) into positive and non-positive values.) A first example of a calculation of a full set of the master integrals with 'elliptic sectors' can be found in Ref. [22], where elliptic functions appear only in two sectors and final results are expressed either in terms of multiple polylogarithms or, for the elliptic sectors, in terms of two and three-fold iterated integrals suitable for numerical evaluation. ${ }^{3}$ Moreover, in Refs. [24,25] a strategy to obtain parametric representations for master integrals applicable also in situations without $\varepsilon$-form was described and illustrated through one-, two- and three-loop examples.

Other examples of calculations of individual Feynman integrals in situations where $\varepsilon$-form is impossible can be found in $[26,27]$ (see also references therein), where results are expressed in

\footnotetext{
${ }^{1}$ See also $[13,14]$ where an algorithm in the case of two and more variables is described and implemented.

${ }^{2}$ Recently, a strict criterion of the existence of an $\varepsilon$-form was presented in Ref. [20].

${ }^{3}$ A similar more recent example can be found in Ref. [23]
} 
terms of elliptic generalizations of polylogarithms [26] or iterated integrals of modular forms [27]. More recent results on elliptic generalization of multiple polylogarithms can be found in Refs. [2831]. However, these new functions do not have the same status as harmonic polylogarithms and multiple polylogarithms, at least in the practical sense, i.e. there are no codes to evaluate them at a given point with a desired precision. Anyway, it looks like we are very far, even in lower loops orders, from answering the following question: 'What is the class of functions which can appear in results for Feynman integrals in situations where $\varepsilon$-form is impossible'?

On the other hand, thinking positively, we may say that knowing a differential system and the corresponding boundary conditions gives almost as much information about Feynman integrals as knowing their explicit expressions in terms of some class of functions. In fact, some properties of the integrals are even more accessible via DE. In particular, singularities of DE provide a way to examine the branching properties of integrals. Numerical values of the integrals can be obtained from a numerical solution ${ }^{4}$ of the differential system. Many computer algebra systems contain tools to solve this task (e.g. NDSolve procedure in Mathematica system). However, there is one complication that does not allow to use these tools immediately. Namely, we would like to keep $\varepsilon$ as a variable and evaluate solutions of DE as series expansions in $\varepsilon$.

The goal of the present paper is to describe an algorithm which enables one to find a solution of a given differential system in the form of an $\varepsilon$-expansion series with numerical coefficients. We describe such an algorithm in the case of Feynman integrals depending on one variable, i.e. with two scales where the variable is introduced as the ratio of these scales. As a proof of concept, we provide a computer code where this algorithm is implemented for a simple example of a family of Feynman integrals where the $\varepsilon$-form is impossible. The general idea behind our approach is to use generalized power series expansions near the singular points of the differential system and solve difference equations for the corresponding coefficients in these expansions. This idea is very well known in mathematics. In high-energy physics, its application to Feynman integrals can be found, for example, in Ref. [34], where three-loop massive vacuum diagrams were evaluated. ${ }^{5}$

In the next section, we present an algorithm to solve difference equations for coefficients of the series expansions at a given singular point. In Section 3, we describe a matching procedure which enables one to connect series expansions at two neighboring points. In Section 4, we describe a computer code based on our algorithm and the matching procedure to evaluate master integrals in a simple four-loop example. Then we conclude with a discussion of perspectives.

\section{Generalized series expansion near a singular point}

Let us have a differential system

$$
\partial_{x} J=M(x, \varepsilon) J
$$

\footnotetext{
${ }^{4}$ Examples of solving DE for Feynman integrals numerically can be found in Refs. [32,33].

${ }^{5}$ Another example, where the general theory of DE was applied for evaluating expansion of two-scale integrals at a given singular point, can be found in Ref. [35]. For this purpose, one can apply various mathematical prescriptions from the theory of DE - see, e.g. Ref. [36], where an algorithm [37] to obtain first terms of expansion near a singular point was applied. An approach similar to Ref. [35] was applied in Ref. [38] to evaluate expansions of solutions of DE at a given singular point by difference equations.
} 
where $J$ is a column-vector of $N$ functions, and $M$ is an $N \times N$ matrix with entries being rational functions ${ }^{6}$ of $x$ and $\varepsilon$. Below we will suppress $\varepsilon$ in the arguments for brevity. We assume that all the singular points of the differential system are regular. Therefor we can reduce the differential system to a local Fuchsian form in any singular point. The general solution has the form

$$
J(x)=U(x) C,
$$

where $C$ is a column of constants, and $U$ is an evolution operator

$$
U(x)=P \exp \left[\int d x M(x)\right]
$$

We want to expand this operator in the vicinity of each singular point. Without loss of generality, let us consider the expansion near $x=0$. It is well known that the expansion has the form

$$
U(x)=\sum_{\lambda \in S} x^{\lambda} \sum_{n=0}^{\infty} \sum_{k=0}^{K_{\lambda}} \frac{1}{k !} C(n+\lambda, k) x^{n} \ln ^{k} x,
$$

where $S$ is a finite set of powers of the form $\lambda=r \varepsilon$ with integer $r, K_{\lambda} \geqslant 0$ is an integer number corresponding to the the maximal power of the logarithm. We have introduced the factor $1 / k$ ! for convenience. Our goal is to determine $S, K_{\lambda}$, and the matrix coefficients $C(n+\lambda, k)$. As to the latter, we are going to determine them via recurrence relations equipped with initial conditions.

Since we assume that the differential system has only regular singular points, we can reduce it at $x=0$ to normalized Fuchsian form [20] by means of rational transformations. For the sake of presentation, we will assume that the system is in global normalized Fuchsian form, i.e.,

$$
M(x)=\frac{A_{0}}{x}+\sum_{k=1}^{s} \frac{A_{k}}{x-x_{k}}
$$

and for any $k=0, \ldots, s$ the matrix $A_{k}$ is free of resonances, i.e. the difference of any two of its distinct eigenvalues is not integer. Note that the $\varepsilon$-form is only one example of normalized Fuchsian form, so we allow for a much wider class of differential systems which seems to be sufficient for any applications in multiloop calculations. In particular, the 'elliptic' cases, as a rule, can easily be reduced to a global normalized Fuchsian form. Besides, it is easy to generalize our algorithm properly if needed.

The operator $U$, Eq. (2.3]), is determined up to a multiplication by a constant matrix from the right. We fix it by the condition

$$
U(x) \stackrel{x \rightarrow 0}{\sim} x^{A_{0}} .
$$

This condition is, strictly speaking, mathematically incorrect when the distance between some eigenvalues of $A_{0}$ is larger than one, but it should be understood as the constraint on the leading terms of the expansion for each distinct eigenvalue. This condition gives us a way to determine $S$, i.e. the set of distinct eigenvalues of $A_{0}$, and $K_{\lambda}$, i.e. the highest power of the logarithm in

\footnotetext{
${ }^{6}$ Typically, $x$ is the dimensionless ratio of two scales for a family of dimensional regularized Feynman integrals depending on two scales.
} 
front of $x^{\lambda}$ in $x^{A_{0}}$ for each $\lambda \in S$, and the leading coefficients $C(\lambda, k)$. We simply determine these parameters by representing

$$
x^{A_{0}}=\sum_{\lambda \in S} x^{\lambda} \sum_{k=0}^{K_{\lambda}} \frac{1}{k !} C(\lambda, k) \ln ^{k} x .
$$

In Ref. [21] an algorithm to solve difference equations for coefficients in (2.4) was presented. The corresponding solutions have a linear growth of the computational complexity with the number of expansion terms.

\section{Matching}

The above considerations enable one to evaluate the evolution operator $(\sqrt{2.3}])$ within the convergence region of the power series (‥4). In order to perform an analytical continuation to the whole complex plane, one may use the same approach for the expansion around other singular points. Suppose that the next singular point closest to the origin is $x=1$. We can construct the evolution operator (2.3]) also in an expansion near this point.

$$
\tilde{U}(x)=P \exp \left[\int d x M(x)\right] .
$$

In general, due to the above mentioned freedom in definition of the evolution operator we have $U(x)=\tilde{U}(x) L$. where $L$ is some constant matrix. If the convergence regions of the power series in $U$ and $\tilde{U}$ overlap, we may fix $L$ by picking some point in the intersection of these regions. E.g. at $x=1 / 2$ we have ${ }^{7} L=\tilde{U}^{-1}(1 / 2) U(1 / 2)$, i.e., finally, in the whole convergence region of $\tilde{U}$ we have $U(x)=\tilde{U}(x) \tilde{U}^{-1}(1 / 2) U(1 / 2)$. Acting in the same way, we may, in principle, extend the definition of $U$ onto the whole complex plane of $x$. In fact, this is a general approach to the analytical continuation of a function defined by a converging power series. In order to reach an arbitrary finite point of the complex plane, we are likely to need also expansions near the regular points (reducible to the considered case by putting $A_{0}=0$ ) and/or Möbius transformations of the variable. In the case where the singularities lie on the real axis and if we are interested in the evaluation of Feynman integrals for real $x$, we can avoid expansions near regular points and rely only on the Möbius transformations. Suppose that we have the following sequence of the singular points $x_{0}<x_{1}<\ldots x_{s}<\infty=x_{s+1}=x_{-1}$. Then for each $0 \leqslant k \leqslant s$ we make the variable change $y_{k}(x)=\frac{a x+b}{c x+d}$ which maps the points $x_{k-1}, x_{k}, x_{k+1}$ to $\mp 1,0, \pm 1$, respectively. It is convenient to choose the sign in such a way that the cuts of the non-integer powers and logarithms appearing in the series expansions coincide with the cuts of the integral.

\section{Implementation}

The four master integrals we evaluate form a basis of the following family of integrals:

$$
\begin{aligned}
F_{a_{1}, \ldots, a_{14}}= & \int \ldots \int \frac{\mathrm{d}^{D} k_{1} \ldots \mathrm{d}^{D} k_{4}\left(k_{1} \cdot p\right)^{a_{6}}\left(k_{2} \cdot p\right)^{a_{7}}\left(k_{3} \cdot p\right)^{a_{8}}\left(k_{4} \cdot p\right)^{a_{9}}}{\left(-k_{1}^{2}\right)^{a_{1}}\left(-k_{2}^{2}\right)^{a_{2}}\left(m^{2}-k_{3}^{2}\right)^{a_{3}}\left(m^{2}-k_{4}^{2}\right)^{a_{4}}\left(m^{2}-\left(\sum k_{i}+p\right)^{2}\right)^{a_{5}}} \\
& \times\left(k_{1} \cdot k_{2}\right)^{a_{10}}\left(k_{1} \cdot k_{3}\right)^{a_{11}}\left(k_{1} \cdot k_{4}\right)^{a_{12}}\left(k_{2} \cdot k_{3}\right)^{a_{13}}\left(k_{2} \cdot k_{4}\right)^{a_{14}},
\end{aligned}
$$

${ }^{7}$ The convergence radius of the power series is equal to the distance to the closest singularity, so $x=1 / 2$ necessarily belongs to the convergence region of the series representation of $U$. We also assume here that it belongs to the convergence region of $\tilde{U}$. 
where $p$ is the external momentum and $m$ is the mass of three lines. They correspond to the generalized sunset graph shown in Fig. W. We introduce $x=p^{2} / m^{2}$.

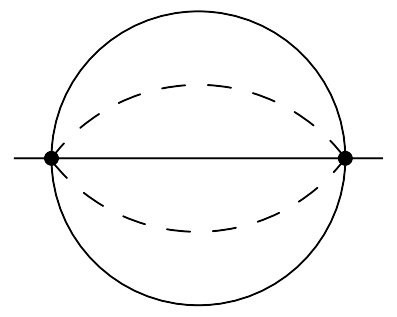

Figure 1: The generalized sunset graph with two massless and three massive lines with the same mass.

There are four master integrals in this family. We choose the following basis:

$$
J_{0}=\left\{F_{1,1,1,1,1,0, \ldots, 0}, F_{1,1,2,1,1,0, \ldots, 0}, F_{1,2,1,1,1,0, \ldots, 0}, F_{1,2,1,1,2,0, \ldots, 0}\right\} .
$$

We derive DE for $J_{0}$ in a straightforward way. When taking derivatives with respect to $x$ one can apply LiteRed $[42,43]$ to do this automatically. The derivatives are then expressed in terms of integrals of the given family. Solving integration by parts relations with an IBP-reduction code ${ }^{8}$, one expresses these derivatives as linear combinations of the primary master integrals and obtains a system of linear DE which has the form (2.

The matrix in the corresponding DE and other entries mentioned in the section, can be can be downloaded from https://bitbucket.org/feynmanintegrals/dess. One uses

$\{\mathrm{M}, \mathrm{T}, \mathrm{Ti}, \mathrm{Mf}\}=<$ "Data/TransformationData";

Here $\mathrm{M}$ is the matrix in the DE for the basis of the chosen primary master integrals (4.2) and $\mathrm{Ti}$ is $T^{-1}$. We turn to the basis $J=T^{-1} \cdot J_{0}$ for which we have the matrix $M_{\mathrm{f}}$ with normalized Fuchsian singularities at any singular point in the corresponding DE (2. d). We have $M_{f}=T^{-1}\left(M \cdot T-\partial_{x} T\right)$. We find the new basis with the help of the algorithm of Ref. [9].

To fix boundary conditions we choose the point $x=0$ where the integrals of the given family become vacuum integrals. To evaluate the four master integrals at $x=0$ we derive onefold MellinBarnes representations for them and obtain the possibility to achieve a high precision for any given coefficient in the $\varepsilon$-expansion. We restricted ourselves to the accuracy of 500 digits but one can increase it to 1000 digits and more.

The singular points are $x_{0}=0, x_{1}=1, x_{2}=9$ and $x_{3}=x_{-1}=\infty$. We solve difference equations for coefficients in series expansions near singular points according to the algorithm described in Section 2. The corresponding results are encoded in a file present in the package:

$\{$ L, cis, cisrule $\}=$ Get ["Data/BoundaryConditions"];

Here $\mathrm{L}$ is a constant matrix (see Section 2) and the list cis defines the required information about the primary masters. The list has the form $\{\operatorname{ci}[1, \varepsilon-1,0], \operatorname{ci}[2,0,0], \operatorname{ci}[3, \varepsilon-1,0], \operatorname{ci}[4,0,0]\}$, where $c i[j, n, k]$ denotes the coefficient in front of $x^{n} \ln ^{k} x$ in $j$-th primary master. The list of

\footnotetext{
${ }^{8}$ In our paper, we use FIRE [39-41] in combination with LiteRed [42,43].
} 
replacement rules cisrule contains this required information which was obtained using different techniques, in particular, Mellin-Barnes representations.

The matching procedure described in the previous section is performed in our example as follows. The variable changes corresponding to the singular points are $f_{0}=x /(2-x), f_{1}=(x-$ $1) /(1+7 x / 9), f_{2}=(9-x) /(7+x), f_{3}=-9 /(2 x-9)$. For example, the first function maps 0 to 0 , 1 to 1 and infinity to -1 . In new coordinates the radius of convergence is equal to 1 , however, the convergence is very slow when approaching the boarder of the convergence domain.

For adjacent regions $i$ and $i+1$ we search the best possible matching point which is such $x$ that it lies between $x_{i}$ and $x_{i+1}$ and that $\left|f_{i}(x)\right|=\left|f_{i+1}(x)\right|$. In our case we result in matching points $\{-3,3(3-2 \sqrt{2}), 3,3(3+2 \sqrt{2})\}$.

The matching points are separating the singular points. We have

$$
-\infty<-3<0<3(3-2 \sqrt{2})<1<3<9<3(3+2 \sqrt{2})<\infty .
$$

Now to obtain the values in a region different from $(-3,3(3-2 \sqrt{2}))$ we have to perform matching by moving in the positive or negative direction. The regions $(3(3-2 \sqrt{2}), 3)$ and $(3(3+2 \sqrt{2}),-3)$ (around infinity) are adjacent and one matching is enough. For the remaining $(3,3(3+2 \sqrt{2})$ ) region one performs two matchings. This procedure is performed automatically in the code DESS.m we provide. The basic function is

DESS [rdatas, $\mathrm{x}, \mathrm{x} 0, \mathrm{oe}, \mathrm{np}]$

It builds the evolution operator near a given point $x_{0}$, where oe is the order in $\varepsilon, \mathrm{np}$ is the required precision, and rdatas contains all the required information about coefficients in expansions at all the singular points in a special format.

The action of this procedure is performed with the help of the following auxiliary functions:

FindLFT $[x,\{k, l, m\}]$

finds the Möbius transformation in $x$ which maps $k, l, m$ to $(-1,0,1)$;

FindMPoint $[x,\{f 1, f 2\}]$

finds the matching point $x$ with $\left|f_{1}(x)\right|=\left|f_{2}(x)\right|$ such that $x$ is between $f_{1}^{-1}(0)$ and $f_{2}^{-1}(0)$;

InverseLFT $[x, f]$

returns the inverse linear transformation function.

The manipulations with series expansion are performed in the auxiliary basis $J$, rather in the primary basis $J_{0}$. For the evaluation of $J_{0}$, one takes into account the relation between the bases and applies the command

$(\mathrm{T} / . \mathrm{x}->\mathrm{x} 0)$.DESS[rdatas, $\mathrm{x}, \mathrm{x} 0, \mathrm{oe}, \mathrm{np}] . \mathrm{L}$. (cis /. cisrule)

to evaluate the set of the primary master integrals (4.2) at the point $x_{0}$ (different from the singular points) in an $\varepsilon$-expansion up to the order oe with the accuracy np.

To test our code we ran our procedure with oe $=15$ and $n p=75$ at the sample points 
$-10,-3 / 2,1 / 3,2 / 3,2,4,12,25$ which lie between the singular and matching points and confirmed our results with the code FIESTA [44]. For example, at $x_{0}=25$, we obtain the following result (shown with a truncation to 10 digits) for the first primary integral:

$$
\begin{aligned}
& -\frac{0.25}{\varepsilon^{4}}+\frac{2.125}{\varepsilon^{3}}-\frac{0.2391337000}{\varepsilon^{2}}-\frac{5.2663306926}{\varepsilon}-185.9464179437+6.5261388472 \mathrm{i} \\
& -(1825.1476432369-48.9550593728 \mathrm{i}) \varepsilon-(8406.8551978029-176.0638485153 \mathrm{i}) \varepsilon^{2} \\
& -(58330.4283767260-401.9617475893 \mathrm{i}) \varepsilon^{3} .
\end{aligned}
$$

In fact, the maximal order of expansion in $\varepsilon$ and the maximal accuracy is determined by the boundary conditions where expansion of boundary vacuum integrals is included up to $\varepsilon^{3}$ with the accuracy of 500 digits. This results in an $\varepsilon$-expansion up to $\varepsilon^{3}$ of our primary master integrals. However, we recommend to set oe $=15$ because high negative powers of $\varepsilon$ appear in calculations. Moreover, we recommend to add the value 25 to the desired precision $\mathrm{np}$, for a similar reason.

One more command of our code is denoted in the same way but has one more argument: DESS [rdatas, $x, f(x), o e, n p, n t]$. It can be used to obtain a required number $n t$ of terms of expansion near a given singular point, i.e. $x_{0} \in\{0,1,9, \infty\}$. For the three finite singular points, one can request an expansion in powers of $f(x)$ which can be any function of the form $f(x)=\left(x-x_{0}\right) /(a x+b)$ different from constant, in particular, $f(x)$ can be $\pm\left(x-x_{0}\right)$. For example, at $x_{0}=9$, the choice $f(x)=9-x$ looks natural and leads to an expansion of the primary master integrals in powers and logarithms of $9-x$ with real coefficients. For the singular point $x_{0}=\infty$, one can choose $f(x)=(a x+b)^{-1}$, e.g., $\pm 1 / x$. Here also the choice $-1 / x$ is natural for the same reason as above. The output of this command (with one more argument) is in the form of a set of replacements $n, j, k \rightarrow \ldots$ which give results for the coefficients $C(n+\varepsilon j, k)$ in Eq. (R.4) in the expansion of the evolution operator near a singular point. A result in the form of Eq. (L. obtained from this result by applying the command

FromCoefficientRules[..., $\left\{x, x^{\wedge} \backslash[\right.$ Epsilon], Log[x] $\left.\}\right]$

Similarly to the evaluation of the master integrals at a given non-singular point, one has to multiply DESS[rdatas, $x, f(x)$, oe, np, nt] by L. (cis /. cisrule) from the right and by an expansion of the transformation matrix $T$ near $x=x_{0}$ from the left and then reexpand the product of all the factors at $x=x_{0}$. The results for the evolution operator given by DESS [rdatas, $\mathrm{x}, \mathrm{f}(\mathrm{x}), \mathrm{oe}, \mathrm{np}, \mathrm{nt}]$ are linear combinations of $\left( \pm\left(x-x_{0}\right)\right)^{n+j \varepsilon}$ so that it is possible to select contributions for specific $j$. For example, one can arrive at results for the naive part of the expansion of the primary master integrals near a given finite singular point by selecting only integer powers. In fact, near $x_{0}=0$ and $x_{0}=1$, we have only Taylor expansions

Let us show that our code can provide results for master integrals for two families of Feynman integrals associated with the same graph of Fig. 1 and defined by (4. (1) but with two different (onshell and threshold) restrictions: $p^{2}=m^{2}$ and $p^{2}=9 m^{2}$, i.e. $x=1$ and $x=9$. In both cases, there are three master integrals which can be chosen as $\left\{F_{1,1,1,1,1,0, \ldots, 0}^{(i)}, F_{1,1,2,1,1,0, \ldots, 0}^{(i)}, F_{1,2,1,1,1,0, \ldots, 0}^{(i)}\right\}$, where $i=1,2$ corresponds to these two families. For both cases, high-precision values for the master integrals can be obtained with the code DESS as it was explained in the previous section. With the boundary conditions with the accuracy of 500 digits supplied with the code, we obtain the accuracy 
of at least 480 digits of the results and find agreement with the analytical results for these master integrals obtained in $[45,46]$. In particular, for the first master integral, the result is

$$
\begin{aligned}
& F_{1,1,1,1,1,0, \ldots, 0}^{(1)}=-\frac{1}{4 \varepsilon^{4}}-\frac{7}{8 \varepsilon^{3}}-\left(\frac{17}{12}+\frac{\pi^{2}}{12}\right) \frac{1}{\varepsilon^{2}}+\left(\frac{\zeta(3)}{3}-\frac{835}{576}-\frac{7 \pi^{2}}{24}\right) \frac{1}{\varepsilon} \\
& +\left(\frac{7 \zeta(3)}{6}-\frac{7379}{6912}-\frac{17 \pi^{2}}{36}-\frac{3 \pi^{4}}{20}\right) \\
& +\left(\frac{17 \zeta(3)}{9}+\frac{127 \pi^{2} \zeta(3)}{9}-\frac{289 \zeta(5)}{5}+\frac{6766055}{82944}-\frac{41539 \pi^{2}}{1728}-\frac{21 \pi^{4}}{40}\right) \varepsilon \\
& +\left(-288 \pi^{2} \operatorname{Li}_{4}\left(\frac{1}{2}\right)-\frac{355325 \zeta(3)}{432}+\frac{889 \pi^{2} \zeta(3)}{18}+\frac{2203 \zeta(3)^{2}}{9}-\frac{2023 \zeta(5)}{10}\right. \\
& -252 \pi^{2} \zeta(3) \log (2)+\frac{1449210865}{995328}-\frac{8822483 \pi^{2}}{20736}-\frac{17 \pi^{4}}{20}+\frac{3877 \pi^{6}}{1890} \\
& \left.-12 \pi^{2} \log ^{4}(2)+12 \pi^{4} \log ^{2}(2)+424 \pi^{2} \log (2)\right) \varepsilon^{2} \\
& +(-5760 \zeta(-6,-1)-5760 \zeta(-6,1)-5760 \zeta(-5,2) \\
& -5760 \zeta(-5,1,1)-5760 \zeta(5,-1,-1)+5760 s_{6} \log (2)-10080 \mathrm{Li}_{4}\left(\frac{1}{2}\right) \zeta(3) \\
& +5184 \pi^{2} \mathrm{Li}_{5}\left(\frac{1}{2}\right)-1008 \pi^{2} \mathrm{Li}_{4}\left(\frac{1}{2}\right)-16960 \mathrm{Li}_{4}\left(\frac{1}{2}\right)+5184 \pi^{2} \mathrm{Li}_{4}\left(\frac{1}{2}\right) \log (2) \\
& +\frac{312867 \zeta(7)}{14}-\frac{100204 \pi^{2} \zeta(5)}{15}-\frac{4913 \zeta(5)}{15}+\frac{15421 \zeta(3)^{2}}{18}+\frac{908 \pi^{4} \zeta(3)}{15} \\
& +\frac{2159 \pi^{2} \zeta(3)}{27}-\frac{77124781 \zeta(3)}{5184}-420 \zeta(3) \log ^{4}(2)+2688 \pi^{2} \zeta(3) \log ^{2}(2) \\
& -7200 \zeta(3)^{2} \log (2)-882 \pi^{2} \zeta(3) \log (2)+\frac{3877 \pi^{6}}{540}+\frac{195233 \pi^{4}}{1728}-\frac{1121725465 \pi^{2}}{248832} \\
& +\frac{182188906799}{11943936}+\frac{864}{5} \pi^{2} \log ^{5}(2)-42 \pi^{2} \log ^{4}(2)-\frac{2120 \log ^{4}(2)}{3}-144 \pi^{4} \log ^{3}(2) \\
& \left.+42 \pi^{4} \log ^{2}(2)-\frac{9328}{3} \pi^{2} \log ^{2}(2)-\frac{14}{3} \pi^{6} \log (2)+7652 \pi^{2} \log (2)\right) \varepsilon^{3}+O\left(\varepsilon^{4}\right),
\end{aligned}
$$

where $\zeta(\ldots)$ are multiple zeta values.

Using our code for the threshold master integrals and applying the pslq algorithm [47] we arrive at the following analytical results for the pole parts in $\varepsilon$ :

$$
\begin{aligned}
& F_{1,1,1,1,1,0, \ldots, 0}^{(2)}=-\frac{1}{4 \varepsilon^{4}}+\frac{1}{8 \varepsilon^{3}}+\frac{\frac{23}{12}-\frac{\pi^{2}}{12}}{\varepsilon^{2}}+\left(\frac{\zeta(3)}{3}+\frac{1493}{576}+\frac{\pi^{2}}{24}\right) \frac{1}{\varepsilon}+\ldots, \\
& F_{1,1,2,1,1,0, \ldots, 0}^{(2)}=\frac{1}{4 \varepsilon^{4}}-\frac{1}{12 \varepsilon^{3}}+\frac{\frac{\pi^{2}}{12}-1}{\varepsilon^{2}}-\left(\frac{\zeta(3)}{3}+\frac{31}{48}+\frac{\pi^{2}}{36}\right) \frac{1}{\varepsilon}+\ldots, \\
& F_{1,2,1,1,1,0, \ldots, 0}^{(2)}=-\frac{1}{4 \varepsilon^{4}}+\frac{2-\frac{\pi^{2}}{12}}{\varepsilon^{2}}+\left(\frac{128 \pi}{3 \sqrt{3}}-\frac{160}{9} \pi \operatorname{Im}\left(\operatorname{Li}_{2}\left(\mathrm{e}^{\mathrm{i} \pi}\right)\right)+\frac{211 \zeta(3)}{9}-\frac{277}{8}\right) \frac{1}{\varepsilon}+\ldots
\end{aligned}
$$

\section{Conclusion}

Our algorithm is oriented at situations where canonical form of the DE is impossible. We have provided a computer implementation of the algorithm in a simple example. This code is similar 
in spirit to the well-known existing codes to evaluate harmonic polylogarithms [15] and multiple polylogarithms [16], where the problem of evaluation reduces to summing up appropriate series. We hope that one can use our algorithm and implement it to evaluate master integrals in situations where an analytic evaluation is problematic.

Of course, one can hardy construct a general algorithm to fix boundary conditions because, usually, the choice of the corresponding point and the way to obtain data for the boundary conditions is done in every situation in a special way. Still we can suggest a format for including information about the boundary conditions for using it in our future package. Anyway, our future package would check if a given system of DE is already in a global normalized Fuchsian form, with singularities on the real axis, and, if this is true, the package would automatically construct the evolution operator in an expansion up to a required order.

\section{Acknowledgments}

V.S. is grateful to Oleg Veretin for useful discussions. The work of A.S. and V.S. was supported by RFBR, grant 17-02-00175A. The work of R. Lee was supported by the grant of the 'Basis' foundation for theoretical physics.

\section{References}

[1] A. V. Kotikov, Phys. Lett. B 254 (1991) 158.

[2] A. V. Kotikov, Phys. Lett. B 267 (1991) 123.

[3] E. Remiddi, Nuovo Cim. A 110 (1997) 1435 [hep-th/9711188].

[4] T. Gehrmann and E. Remiddi, Nucl. Phys. B 580 (2000) 485 [hep-ph/9912329].

[5] T. Gehrmann and E. Remiddi, Nucl. Phys. B 601 (2001) 248 [hep-ph/0008287].

[6] T. Gehrmann and E. Remiddi, Nucl. Phys. B 601 (2001) 287 [hep-ph/0101124].

[7] J. M. Henn, Phys. Rev. Lett. 110 (2013) 25, 251601 [arXiv:1304.1806 [hep-th]].

[8] K. G. Chetyrkin and F. V. Tkachov, Nucl. Phys. B 192 (1981) 159.

[9] R. N. Lee, JHEP 1504 (2015) 108 [arXiv:1411.0911 [hep-ph]].

[10] O. Gituliar and V. Magerya, PoS LL 2016 (2016) 030 [arXiv:1607.00759 [hep-ph]].

[11] O. Gituliar and V. Magerya, Comput. Phys. Commun. 219 (2017) 329 [arXiv:1701.04269 [hep-ph]].

[12] M. Prausa, Comput. Phys. Commun. 219 (2017) 361 [arXiv:1701.00725 [hep-ph]].

[13] C. Meyer, JHEP 1704 (2017) 006 [arXiv:1611.01087 [hep-ph]].

[14] C. Meyer, arXiv:1705.06252 [hep-ph].

[15] E. Remiddi and J. A. M. Vermaseren, Int. J. Mod. Phys. A 15 (2000) 725 [hep-ph/9905237].

[16] A. B. Goncharov, Math. Res. Lett. 5 (1998) 497-516 [arXiv:1105.2076].

[17] D. Maitre, Comput. Phys. Commun. 174 (2006) 222, [hep-ph/0507152].

[18] J. Vollinga and S. Weinzierl, Comput. Phys. Commun. 167 (2005) 177 [hep-ph/0410259]. 
[19] C. W. Bauer, A. Frink and R. Kreckel, J. Symb. Comput. 33 (2000) 1 [cs/0004015 [cs-sc]].

[20] R. N. Lee and A. A. Pomeransky, arXiv:1707.07856 [hep-th].

[21] R. N. Lee, A. V. Smirnov and V. A. Smirnov, arXiv:1709.07525 [hep-ph].

[22] R. Bonciani, V. Del Duca, H. Frellesvig, J. M. Henn, F. Moriello and V. A. Smirnov, JHEP 1612 (2016) 096 [arXiv:1609.06685 [hep-ph]].

[23] M. Becchetti and R. Bonciani, arXiv:1712.02537 [hep-ph].

[24] A. Primo and L. Tancredi, Nucl. Phys. B 916 (2017) 94 [arXiv:1610.08397 [hep-ph]].

[25] A. Primo and L. Tancredi, Nucl. Phys. B 921 (2017) 316 [arXiv:1704.05465 [hep-ph]].

[26] L. Adams, C. Bogner, A. Schweitzer and S. Weinzierl, J. Math. Phys. 57 (2016) 122302 [arXiv:1607.01571 [hep-ph]].

[27] L. Adams and S. Weinzierl, arXiv:1704.08895 [hep-ph].

[28] E. Remiddi and L. Tancredi, arXiv:1709.03622 [hep-ph].

[29] M. Hidding and F. Moriello, arXiv:1712.04441 [hep-ph].

[30] J. Broedel, C. Duhr, F. Dulat and L. Tancredi, arXiv:1712.07089 [hep-th].

[31] J. Broedel, C. Duhr, F. Dulat and L. Tancredi, arXiv:1712.07095 [hep-ph].

[32] M. Czakon, Phys. Lett. B 664 (2008) 307 [arXiv:0803.1400 [hep-ph]].

[33] P. Bärnreuther, M. Czakon and P. Fiedler, JHEP 1402 (2014) 078 [arXiv:1312.6279 [hep-ph]].

[34] B. A. Kniehl, A. F. Pikelner and O. L. Veretin, JHEP 1708 (2017) 024 [arXiv:1705.05136 [hep-ph]].

[35] R. Mueller and D. G. Öztürk, JHEP 1608 (2016) 055 [arXiv:1512.08570 [hep-ph]].

[36] J. M. Henn, A. V. Smirnov and V. A. Smirnov, JHEP 1612 (2016) 144 [arXiv:1611.06523 [hep-ph]].

[37] W. Wasow, “Asymptotic expansions for ordinary differential equations”, Interscience Publishers John Wiley \& Sons, Inc., New York-London-Sydney, Pure and Applied Mathematics, Vol. XIV, 1965.

[38] K. Melnikov, L. Tancredi and C. Wever, JHEP 1611 (2016) 104 [arXiv:1610.03747 [hep-ph]].

[39] A. V. Smirnov, JHEP 0810 (2008) 107 [arXiv:0807.3243 [hep-ph]].

[40] A. V. Smirnov and V. A. Smirnov, Comput. Phys. Commun. 184 (2013) 2820 [arXiv:1302.5885 [hep-ph]].

[41] A. V. Smirnov, Comput. Phys. Commun. 189 (2015) 182 [arXiv:1408.2372 [hep-ph]].

[42] R. N. Lee, arXiv:1212.2685 [hep-ph].

[43] R. N. Lee, J. Phys. Conf. Ser. 523 (2014) 012059 [arXiv:1310.1145 [hep-ph]].

[44] A. V. Smirnov, Comput. Phys. Commun. 204 (2016) 189 [arXiv:1511.03614 [hep-ph]].

[45] P. Marquard, A. V. Smirnov, V. A. Smirnov and M. Steinhauser, Phys. Rev. Lett. 114 (2015) no.14, 142002 [arXiv:1502.01030 [hep-ph]].

[46] P. Marquard, A. V. Smirnov, V. A. Smirnov, M. Steinhauser and D. Wellmann, Phys. Rev. D 94 (2016) no.7, 074025 [arXiv:1606.06754 [hep-ph]].

[47] H. R. P. Ferguson, D. H. Bailey, and S. Arno, Analysis of PSLQ, an integer relation finding algorithm, Math. Comput. 68 (1999) 351-369. 